\title{
Strong-coupling theory of condensate-mediated superconductivity in two-dimensional materials
}

\author{
Meng Sun $\odot,{ }^{1}$ A. V. Parafilo $\odot,{ }^{1}$ V. M. Kovalev $\odot,{ }^{2,3}$ and I. G. Savenko $\circledast^{1,2,4}$ \\ ${ }^{1}$ Center for Theoretical Physics of Complex Systems, Institute for Basic Science (IBS), Daejeon 34126, Korea \\ ${ }^{2}$ A.V. Rzhanov Institute of Semiconductor Physics, Siberian Branch of Russian Academy of Sciences, Novosibirsk 630090, Russia \\ ${ }^{3}$ Department of semiconductor devices and microelectronics, Novosibirsk State Technical University, Novosibirsk 630073, Russia \\ ${ }^{4}$ Basic Science Program, Korea University of Science and Technology (UST), Daejeon 34113, Korea
}

(Received 25 March 2021; revised 21 July 2021; accepted 22 July 2021; published 19 August 2021)

\begin{abstract}
We develop a strong-coupling theory of Bose-Einstein condensate-mediated superconductivity in a hybrid system, which consists of a two-dimensional electron gas with either: (i) parabolic spectrum or (ii) relativistic Dirac spectrum in the vicinity of a two-dimensional solid-state condensate of indirect excitons. The Eliashberg equations are derived, and the expressions for the electron pairing self-energy due to the exchange interaction between electrons mediated by a single Bogoliubov excitation (a bogolon) and the bogolon pairs are found. Furthermore, we find the superconducting order parameter and estimate the critical temperature of the superconducting transition. The critical temperature reveals its linear dependence on the dimensionless coupling constant. It is shown that the bogolon-pair-mediated interaction is the dominant mechanism of electron pairing in hybrid systems in both the weak- and the strong-coupling regimes. We calculate the effective bogolon-electron interaction constant for both parabolic and linear electron dispersions and examine the dependence of the critical temperature of the superconducting transition on exciton condensate density.
\end{abstract}

DOI: 10.1103/PhysRevResearch.3.033166

\section{INTRODUCTION}

The first microscopic description of the superconducting (SC) state belongs to the celebrated Bardeen-CopperSchrieffer (BCS) theory [1-4], which explains the emergence of the SC gap and allows one to estimate the critical temperature of SC transition $T_{c}$. Later, Green's functions formalism-based Migdal-Eliashberg (later Eliashberg) theory was developed [5-7]. It provides a more rigorous and accurate basis for the estimation of $T_{c}$ and allows one to account for the Coulomb repulsion between electrons. Generally, by employing McMillan's approach [8], the formation of Cooper pairs by electron-phonon interaction can be solely determined by the effective electron-phonon coupling strength $\lambda$. As compared with the BCS theory, which only works in the weakcoupling regime (of $\lambda \ll 1$ ), the Eliashberg theory is more general. In particular, it avoids the Debye frequency cutoff, thus, allowing to extend the estimations to the strong coupling regime (of $\lambda$ close to or larger than unity).

The price to pay is that the analytical and numerical calculations by the Eliashberg theory are usually more complicated than the ones by the BCS. It requires the solution of multiple coupled equations, which is often tricky, and some assumptions are in order. Since the 1960s, various numerical approaches implying different special

Published by the American Physical Society under the terms of the Creative Commons Attribution 4.0 International license. Further distribution of this work must maintain attribution to the author(s) and the published article's title, journal citation, and DOI. assumptions and simplifications have been developed in order to find the approximate solutions of the Eliashberg equations. Very recently, there have been suggested powerful density functional-based techniques (called the density functional theory for superconductors) [9-11]. In general, the phononmediated superconductivity is well studied, although there still exist many open questions, in particular, regarding the SC transition in novel two-dimensional (2D) materials and their stacks due to phonon-mediated pairing.

However, electron-phonon interaction is not the only possible route to the formation of Cooper pairs. Among others, there have been reported several proposals for exciton [12-14], exciton-polariton [15-17], and cavity-photonmediated [18] coupling mechanisms between electrons. In our recent works $[19,20]$, we have developed a BCS-like theory for the indirect exciton condensate-mediated superconductivity in hybrid systems consisting of layers of a (Bose-condensed) exciton gas and a 2D electron gas or graphene. Employing the Bogoliubov theory, we considered quasiparticle excitations above the exciton condensate called bogolons. They interact with the electron gas via Coulomb forces, which distinguishes this problem from the phonon-assisted electron-electron interaction in conventional superconductors.

However, all the proposals listed above are based on the BCS-like approach. Thus, the weak effective coupling strength between electrons and phonons (or other particles, such as bogolons) is assumed, which means that $\lambda$ should, strictly speaking, be small. In this paper, we build a strongcoupling theory for bogolon-mediated superconducting in hybrid Bose-Fermi systems - an intense area of research [15,21-25]. By employing the Green's-functions technique, 
we calculate the electron-bogolon coupling strength $\lambda$ and give an estimation for the critical temperature of SC transition. The bosonic subsystem which we consider here is an indirect exciton gas. It can be substituted by other quasicondensates, such as direct exciton gas or exciton-polariton condensates, which have been predicted [26-31] and studied experimentally [32-35]. The condensation in these systems has been reported at relatively high temperatures, even approaching room temperature.

The paper is organized as follows. In Sec. II, we introduce the Hamiltonian of bogolon-electron interaction. In Sec. III, we build the bogolon-mediated Eliashberg theory and derive the Eliashberg spectral function. Furthermore, in Sec. IV, we calculate the electron-bogolon coupling constant $\lambda$ and estimate the critical temperature by considering electrons with parabolic and linear dispersions. Finally, in Sec. IV we summarize the results.

\section{SINGLE-BOGOLON AND BOGOLON- PAIR-MEDIATED INTERACTION}

Let us consider a hybrid system in which a 2D layer of electron gas (2DEG) and a layer of a Bose-Einstein condensate (BEC) are in the vicinity of each other in the $z$ direction (we consider the same setup as in works $[19,20,36]$ ). The bosonic subsystem is represented by indirect excitons where the formation of a BEC has been recently reported $[35,37]$. Indirect excitons consist of electrons and holes residing in $n$ - and $p$-doped layers which are separated in the $z$ direction. These layers can be made of, e.g., $\mathrm{MoS}_{2}$ or $\mathrm{WSe}_{2}$ materials separated by several layers of hexagonal boron nitride (hBN) [35,38]. The 2D electron gas (described by the field operator $\Psi_{\sigma}(\mathbf{r})$ with the position vector $\mathbf{r}$ and the spin $\sigma=\{\uparrow, \downarrow\})$ and the exciton gas [described by the field operator $\Phi(\mathbf{r})$ ] layers are also spatially separated by a hBN layer. The electrons and indirect excitons are coupled by the Coulomb interaction [22,39],

$$
\mathcal{H}=\sum_{\sigma} \int d \mathbf{r} \int d \mathbf{R} \Psi_{\sigma}^{\dagger}(\mathbf{r}) \Psi_{\sigma}(\mathbf{r}) g(\mathbf{r}-\mathbf{R}) \Phi^{\dagger}(\mathbf{R}) \Phi(\mathbf{R}),
$$

where $\mathbf{R}$ is the in-plane position vector of the exciton center-of-mass motion. We consider the case when the most of excitons are being in the ground state (in BEC). Then, in the weakly interacting regime, one can write the exciton field operator as $\Phi(\mathbf{R})=\sqrt{n_{c}}+\varphi(\mathbf{R})$, where $n_{c}$ is the condensate density and $\varphi(\mathbf{R})$ is the field operator for noncondensed excitons. Applying the Fourier and the Bogoliubov transformations, from (1), we can find the Hamiltonian for the one-bogolon (1b) and bogolon-pair-mediated (2b) interactions $[19,20,36,40]$ (putting $\hbar=k_{B}=1$ below for simplicity and restoring these constants later),

$\mathcal{H}_{1}=\frac{\sqrt{n_{c}}}{L} \sum_{\mathbf{k} \mathbf{p} \sigma} g_{\mathbf{p}}\left[\left(v_{\mathbf{p}}+u_{-\mathbf{p}}\right) b_{-\mathbf{p}}^{\dagger}+\left(v_{-\mathbf{p}}+u_{\mathbf{p}}\right) b_{\mathbf{p}}\right] c_{\mathbf{k}+\mathbf{p}, \sigma}^{\dagger} c_{\mathbf{k}, \sigma}$,

$$
\begin{aligned}
\mathcal{H}_{2}= & \frac{1}{L^{2}} \sum_{\mathbf{k p q} \sigma} g_{\mathbf{p}}\left[u_{\mathbf{q}-\mathbf{p}} u_{\mathbf{q}} b_{\mathbf{q}-\mathbf{p}}^{\dagger} b_{\mathbf{q}}+u_{\mathbf{q}-\mathbf{p}} v_{\mathbf{q}} b_{\mathbf{q}-\mathbf{p}}^{\dagger} b_{-\mathbf{q}}^{\dagger}\right. \\
& \left.+v_{\mathbf{q}-\mathbf{p}} u_{\mathbf{q}} b_{-\mathbf{q}+\mathbf{p}} b_{\mathbf{q}}+v_{\mathbf{q}-\mathbf{p}} v_{\mathbf{q}} b_{-\mathbf{q}+\mathbf{p}} b_{-\mathbf{q}}^{\dagger}\right] c_{\mathbf{k}+\mathbf{p}, \sigma}^{\dagger} c_{\mathbf{k}, \sigma},
\end{aligned}
$$

where $g_{\mathbf{p}}$ is the Fourier image of electron-exciton interaction; $c_{\mathbf{p}, \sigma}$ and $b_{\mathbf{p}}$ are the annihilation operators for the electrons and bogolons, respectively. The Bogoliubov coefficients are defined as [41]

$$
\begin{aligned}
u_{\mathbf{p}}^{2} & =1+v_{\mathbf{p}}^{2}=\frac{1}{2}\left\{1+\left[1+\left(\frac{M s^{2}}{\omega_{\mathbf{p}}}\right)^{2}\right]^{1 / 2}\right\}, \\
u_{\mathbf{p}} v_{\mathbf{p}} & =-\frac{M s^{2}}{2 \omega_{\mathbf{p}}},
\end{aligned}
$$

where $M$ is the exciton effective mass, $s=\sqrt{\kappa n_{c} / M}$ is the sound velocity, $\kappa=e_{0}^{2} d / \epsilon_{0} \epsilon$ is the exciton-exciton interaction strength in the reciprocal space, $e_{0}$ is the electron charge, $\epsilon$ is the dielectric constant, $\epsilon_{0}$ is the dielectric permittivity, and $\omega_{p}=s p\left(1+p^{2} \xi_{h}^{2}\right)^{1 / 2}$ is the spectrum of bogolons with the healing length $\xi_{h}=1 / 2 M s$.

To find an analytic form of the electron-exciton interaction, we disregard the peculiarities of the exciton internal motion (relative motion of the electron and hole in the exciton). In monolayers of transition-metal dichalcogenides, the exciton binding energy is usually very large: It might even exceed room temperature. Thus, the assumption that an individual exciton is in its ground state with respect to its relative electron-hole motion is reasonable, and only the exciton center-of-mass motion plays an important role. Then, the electron-exciton interaction in direct space reads

$$
g(\mathbf{r}-\mathbf{R})=\frac{e_{0}^{2}}{4 \pi \epsilon_{0} \epsilon}\left(\frac{1}{r_{e e}}-\frac{1}{r_{e h}}\right),
$$

with $r_{e e}=\sqrt{l^{2}+(\mathbf{r}-\mathbf{R})^{2}}$ and $r_{e h}=\sqrt{(l+d)^{2}+(\mathbf{r}-\mathbf{R})^{2}}$; here, $d$ is an effective size of the boson, which is equal to the distance between the $n$ - and $p$-doped layers in the case of an indirect exciton condensate, and $l$ is the separation between the $2 \mathrm{DEG}$ and the BEC. Performing the Fourier transformation, we find

$$
g_{p}=\frac{e_{0}^{2}\left(1-e^{-p d}\right) e^{-p l}}{2 \epsilon_{0} \epsilon p} \approx \frac{e_{0}^{2} d}{2 \epsilon_{0} \epsilon} .
$$

We will consider the contact-interaction case, where $p d \ll 1$ and $p l \ll 1$ for simplicity.

Using the conventional perturbation theory, one may argue that $\mathcal{H}_{1} \gg \mathcal{H}_{2}$ since the density of noncondensed particles is a small quantity as compared with the condensate density, i.e., $\sqrt{n_{c}} b_{\mathbf{p}}^{\dagger} \gg b_{\mathbf{p}}^{\dagger} b_{\mathbf{p}}$. However, with the Bogoliubov coefficients, the summation such as $v_{\mathbf{p}}+u_{-\mathbf{p}}$ in Eq. (2) can drastically change the situation. In the long-wavelength limit, $\xi_{h} p \ll$ 1 , which we consider in this paper, one has $\omega_{p} \approx s p$ and $u_{\mathbf{p}} \approx-v_{\mathbf{p}} \approx \sqrt{M s / p}$. Then, $v_{\mathbf{p}}+u_{\mathbf{p}} \rightarrow 0$ whereas $u_{\mathbf{p}} v_{\mathbf{p}} \rightarrow$ $\infty$. These estimations give a hunch that the contribution of $\mathcal{H}_{2}$ should surpass $\mathcal{H}_{1}$.

\section{THE ELIASHBERG EQUATIONS}

We will work in the framework of the Nambu-Gor'kov formalism $[42,43]$. First, let us introduce the two-component field operator,

$$
\boldsymbol{\Psi}_{\mathbf{k}}=\left(\begin{array}{c}
c_{\mathbf{k} \uparrow} \\
c_{-\mathbf{k} \downarrow}^{\dagger}
\end{array}\right), \quad \boldsymbol{\Psi}_{\mathbf{k}}^{\dagger}=\left(\begin{array}{cc}
c_{\mathbf{k} \uparrow}^{\dagger} & c_{-\mathbf{k} \downarrow}
\end{array}\right),
$$


where $c_{\mathbf{k} \sigma}$ is the annihilation operator of an electron with the momentum $\mathbf{k}$ and spin $\sigma$. Then, the generalized Green's function in the $(2 \times 2)$-matrix form reads

$$
\hat{G}(\mathbf{k}, \tau)=-\left\langle T_{\tau} \boldsymbol{\Psi}_{\mathbf{k}}(\tau) \boldsymbol{\Psi}_{\mathbf{k}}^{\dagger}\right\rangle
$$

or using (8), we can write it as

$$
\hat{G}(\mathbf{k}, \tau)=-\left(\begin{array}{cc}
\left\langle T_{\tau} c_{\mathbf{k} \uparrow}(\tau) c_{\mathbf{k} \uparrow}^{\dagger}\right\rangle & \left\langle T_{\tau} c_{\mathbf{k} \uparrow}(\tau) c_{-\mathbf{k} \downarrow}\right\rangle \\
\left\langle T_{\tau} c_{-\mathbf{k} \downarrow}^{\dagger}(\tau) c_{-\mathbf{k} \downarrow}^{\dagger}\right\rangle & \left\langle T_{\tau} c_{-\mathbf{k} \downarrow}^{\dagger}(\tau) c_{-\mathbf{k} \downarrow}\right\rangle
\end{array}\right) .
$$

The diagonal terms in Eq. (10) represent the standard Green's functions of electron quasiparticles, whereas the off-diagonal terms are the Gor'kov's anomalous Green's functions. Performing the Fourier transform from the imaginary-time domain to the Matsubara frequency representation, we have

$$
\hat{G}\left(\mathbf{k}, i p_{k}\right)=\left(\begin{array}{cc}
\mathcal{G}\left(\mathbf{k}, i p_{k}\right) & \mathcal{F}\left(\mathbf{k}, i p_{k}\right) \\
\mathcal{F}^{*}\left(\mathbf{k}, i p_{k}\right) & -\mathcal{G}\left(-\mathbf{k},-i p_{k}\right)
\end{array}\right) .
$$

To define the bogolons' Green's function, we introduce the notation $A_{\mathbf{p}}=u_{\mathbf{p}} b_{\mathbf{p}}+v_{\mathbf{p}} b_{-\mathbf{p}}^{\dagger}$. Then, the normal and anomalous free Green's functions of the bogolons read $\mathcal{D}(\mathbf{p}, \tau)=$ $-\left\langle T_{\tau} A_{\mathbf{p}}(\tau) A_{\mathbf{p}}^{\dagger}\right\rangle$ and $\mathcal{A}(\mathbf{p}, \tau)=-\left\langle T_{\tau} A_{\mathbf{p}}(\tau) A_{-\mathbf{p}}\right\rangle$, respectively. Switching to the Matsubara frequency domain, we find

$$
\begin{aligned}
& \mathcal{D}\left(\mathbf{p}, i \omega_{n}\right)=\frac{u_{\mathbf{p}}^{2}}{i \omega_{n}-\omega_{\mathbf{p}}}-\frac{v_{\mathbf{p}}^{2}}{i \omega_{n}+\omega_{\mathbf{p}}}, \\
& \mathcal{A}\left(\mathbf{p}, i \omega_{n}\right)=\frac{u_{\mathbf{p}} v_{\mathbf{p}}}{i \omega_{n}-\omega_{\mathbf{p}}}-\frac{u_{\mathbf{p}} v_{\mathbf{p}}}{i \omega_{n}+\omega_{\mathbf{p}}} .
\end{aligned}
$$

In the long-wavelength limit $p \xi_{h} \ll 1$, the Bogoliubov coefficients (4) and (5) read $u_{\mathbf{p}} \approx-v_{\mathbf{p}}$, and, consequently, $\mathcal{D}\left(\mathbf{p}, i \omega_{n}\right) \approx-\mathcal{A}\left(\mathbf{p}, i \omega_{n}\right)$. Using the operators $A_{\mathbf{p}}$ and $A_{\mathbf{p}}^{\dagger}$, the bogolon-mediated interaction with the electronic subsystem given in Eqs. (2) and (3) reads

$$
\begin{aligned}
& \mathcal{H}_{1}=\frac{\sqrt{n_{c}}}{L} \sum_{\mathbf{k p} \sigma} g_{\mathbf{p}}\left(A_{\mathbf{p}}+A_{-\mathbf{p}}^{\dagger}\right) c_{\mathbf{k}+\mathbf{p}, \sigma}^{\dagger} c_{\mathbf{k}, \sigma}, \\
& \mathcal{H}_{2}=\frac{1}{L^{2}} \sum_{\mathbf{k p q} \sigma} g_{\mathbf{p}}\left(A_{\mathbf{q}-\mathbf{p}}^{\dagger} A_{\mathbf{q}}\right) c_{\mathbf{k}+\mathbf{p}, \sigma}^{\dagger} c_{\mathbf{k}, \sigma} .
\end{aligned}
$$

Henceforth, we perform a perturbation theory expansion over the weak interacting terms Eqs. (14) and (15) in order to obtain the Dyson equation for the electron Green's function. The general solution of the Dyson equation reads

$$
\hat{G}^{-1}\left(\mathbf{k}, i p_{k}\right)=\hat{G}_{0}^{-1}\left(\mathbf{k}, i p_{k}\right)-\hat{\Sigma}\left(\mathbf{k}, i p_{k}\right),
$$

where

$$
\hat{G}_{0}^{-1}\left(\mathbf{k}, i p_{k}\right)=i p_{k} \sigma_{0}-\xi_{\mathbf{k}} \sigma_{3}
$$

is the unperturbed Green's function, $\sigma_{\nu=0-3}$ are the Pauli matrices, and $\xi_{\mathbf{k}}$ is the electron dispersion measured with respect to the chemical potential $\xi_{\mathbf{k}}=\varepsilon_{\mathbf{k}}-\mu$.

Following the steps of the standard Eliashberg theory, we separate the electron self-energy into two terms: the usual Coulomb contribution $\hat{\Sigma}_{c}$ and the electron-bogolon contribution $\hat{\Sigma}_{e b}$ (in full analogy with the electron-phonon contribution).

The Coulomb contribution is given by

$$
\hat{\Sigma}_{c}\left(\mathbf{k}, i p_{n}\right)=-\frac{1}{\beta} \sum_{\mathbf{p}, m} \sigma_{3} \hat{G}^{o d}\left(\mathbf{p}, i p_{m}\right) \sigma_{3} V(\mathbf{k}-\mathbf{p}),
$$

where $V(\mathbf{k}-\mathbf{p})$ are the matrix elements of static screened Coulomb interaction between the electronic states $\mathbf{k}$ and $\mathbf{p}$, and $\hat{G}^{o d}$ is the off-diagonal component of the Green's function [4]. The self-energies of the electron-bogolon $\left(\hat{\Sigma}_{1 b}\right)$ and the electron-bogolon-pair $\left(\hat{\Sigma}_{2 b}\right)$ interaction can be calculated by the Dyson equation up to first order, in accordance with the diagrams presented in Fig. 1,

$$
\begin{gathered}
\hat{\Sigma}_{1 b}\left(\mathbf{k}, i p_{k}\right)=\sum_{\mathbf{p}, m} \frac{n_{c} g_{\mathbf{p}}^{2}}{L^{2} \beta} \sigma_{3} \hat{G}\left(\mathbf{k}-\mathbf{p}, i p_{k}-i \omega_{m}\right) \sigma_{3}\left[2 \mathcal{A}\left(\mathbf{p}, i \omega_{m}\right)+\mathcal{D}\left(\mathbf{p}, i \omega_{m}\right)+\mathcal{D}\left(-\mathbf{p},-i \omega_{m}\right)\right] \\
\hat{\Sigma}_{2 b}\left(\mathbf{k}, i p_{k}\right)=\sum_{\mathbf{p}, \mathbf{q}}^{m, n} \frac{g_{\mathbf{p}}^{2}}{L^{4} \beta^{2}} \sigma_{3} \hat{G}\left(\mathbf{k}-\mathbf{p}, i p_{k}-i \omega_{m}-i \omega_{n}\right) \sigma_{3}\left[\mathcal{A}\left(\mathbf{q}-\mathbf{p}, i \omega_{m}\right) \mathcal{A}\left(\mathbf{q}, i \omega_{n}\right)+\mathcal{D}\left(\mathbf{q}-\mathbf{p},-i \omega_{m}\right) \mathcal{D}\left(\mathbf{q}, i \omega_{n}\right)\right],
\end{gathered}
$$

where $p_{k}$ and $\omega_{n}$ are the Matsubara frequencies of the fermions and bosons, respectively. Then (in the long-wavelength limit) we see that $\hat{\Sigma}_{1 b} \rightarrow 0$ because the normal and anomalous Green's functions of the bogolon cancel each other out.

For the $2 \mathrm{~b}$ processes it is not the case, and we introduce the polarization operator $\mathcal{P}\left(\mathbf{p}, i \omega_{n}\right)$, which reads

$$
\begin{aligned}
\mathcal{P}\left(\mathbf{p}, i \omega_{m}\right) & =-\frac{2}{\beta L^{4}} \sum_{\mathbf{q}, n} \mathcal{A}\left(\mathbf{q}-\mathbf{p}, i \omega_{n}+i \omega_{m}\right) \mathcal{A}\left(\mathbf{q}, i \omega_{n}\right) \\
& =-\frac{M^{2} s^{4}}{2 L^{4}} \sum_{\mathbf{q}} \frac{1}{\omega_{\mathbf{q}-\mathbf{p}} \omega_{\mathbf{q}}}\left[\left(\frac{N_{\mathbf{q}-\mathbf{p}}-N_{\mathbf{q}}}{i \omega_{m}+\omega_{\mathbf{q}}-\omega_{\mathbf{q}-\mathbf{p}}}-\frac{N_{\mathbf{q}-\mathbf{p}}-N_{\mathbf{q}}}{i \omega_{m}-\omega_{\mathbf{q}}+\omega_{\mathbf{q}-\mathbf{p}}}\right)+\left(\frac{N_{\mathbf{q}}+N_{\mathbf{q}-\mathbf{p}}+1}{i \omega_{m}+\omega_{\mathbf{q}}+\omega_{\mathbf{q}-\mathbf{p}}}-\frac{N_{\mathbf{q}}+N_{\mathbf{q}-\mathbf{p}}+1}{i \omega_{m}-\omega_{\mathbf{q}}-\omega_{\mathbf{q}-\mathbf{p}}}\right)\right] .
\end{aligned}
$$

In this paper, we will restrict ourselves to the case when the contribution of $N_{\mathbf{q}}$ terms is negligible. As we have shown in earlier works $[19,20]$, the $N_{\mathbf{q}}$-containing correction only results in quantitative difference (more precisely, it results in an increase in the critical temperature of the SC transition), and it is negligible when the size of the condensate is small. 


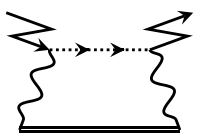

(a)

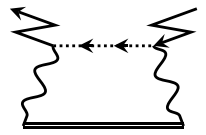

(b)

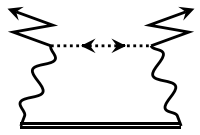

(c)

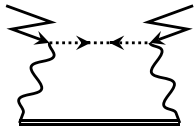

(d)

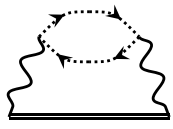

(e)

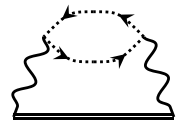

(f)

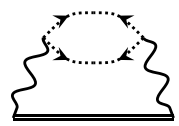

(g)

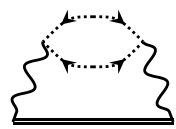

(h)

FIG. 1. Electron self-energy diagrams. The double solid lines stand for the Green's functions of an electron $\hat{G}$ in Eq. (10). The zigzag lines denote the condensate particles. (Thus, each zigzag line gives the factor $\sqrt{n_{c}}$ ). The dotted lines represent bogolons (the particles excited from the condensate). The wiggly lines stand for the electron-exciton interaction $g_{\mathbf{p}}$. Panels (a)-(d) correspond to the 1b process [Eq. (19)], and panels (e)-(h) correspond to the $2 \mathrm{~b}$ process [Eq. (20)]. Physically, the diagrams in (a)-(d) describe the excitation of a condensate particle to a noncondensed state by a moving electron, whereas (e)-(h) describe the condensate polarization due to the moving electron.

Then, the polarization operator simplifies to

$$
\mathcal{P}^{0}\left(\mathbf{p}, i \omega_{m}\right)=\frac{-\kappa^{2} n_{c}^{2}}{L^{4}} \sum_{\mathbf{q}} \frac{1}{\omega_{\mathbf{q}-\mathbf{p}} \omega_{\mathbf{q}}} \frac{\omega_{\mathbf{q}}+\omega_{\mathbf{q}-\mathbf{p}}}{\omega_{m}^{2}+\left(\omega_{\mathbf{q}}+\omega_{\mathbf{q}-\mathbf{p}}\right)^{2}},
$$

and the self-energy can be rewritten in the form,

$$
\begin{aligned}
\hat{\Sigma}_{2 b}\left(\mathbf{k}, i p_{k}\right)= & \frac{-1}{\beta} \sum_{\mathbf{p}, n} g_{\mathbf{k}-\mathbf{p}}^{2} \mathcal{P}^{0}\left(\mathbf{k}-\mathbf{p}, i p_{k}-i p_{n}\right) \\
& \times \sigma_{3} \hat{G}\left(\mathbf{p}, i p_{m}\right) \sigma_{3} .
\end{aligned}
$$

We have already demonstrated, that the self-energy contribution due to the 2 b process is dominant $\hat{\Sigma}_{2 b} \gg \hat{\Sigma}_{1 b}$. We want to note that all other terms, such as three- and four- (and more) bogolon-mediated processes give smaller contributions since all these terms emerge in the perturbative expansion where the small parameter is electron-exciton interaction strength $g_{\mathbf{p}}$. The terms $\hat{\Sigma}_{1 b(2 b)}$ are of the same order $\sim g_{\mathbf{p}}^{2}$, which is the leading order of the expansion.

To proceed further, it is a common practice to decompose the matrix self-energy and rewrite it as a linear combination of Pauli matrices with scalar functions as coefficients [44],

$$
\begin{aligned}
\hat{\Sigma}\left(\mathbf{k}, i p_{k}\right)= & i p_{k}\left[1-Z\left(\mathbf{k}, i p_{k}\right)\right] \sigma_{0}+\chi\left(\mathbf{k}, i p_{k}\right) \sigma_{3} \\
& +\phi\left(\mathbf{k}, i p_{k}\right) \sigma_{1}+\bar{\phi}\left(\mathbf{k}, i p_{k}\right) \sigma_{2},
\end{aligned}
$$

where $Z\left(\mathbf{k}, i p_{k}\right)$ is the mass renormalization function, $\chi\left(\mathbf{k}, i p_{k}\right)$ is the energy shift, $\phi\left(\mathbf{k}, i p_{k}\right)$ and $\bar{\phi}\left(\mathbf{k}, i p_{k}\right)$ are the order parameters. By the gauge transformation [4], we can set the order parameter $\bar{\phi}$ to zero. Then, using (16) and (17), the Green's function reads

$$
\begin{aligned}
\hat{G}\left(\mathbf{k}, i p_{k}\right)= & -\frac{1}{\Theta\left(\mathbf{k}, i p_{k}\right)}\left\{i p_{k} Z\left(\mathbf{k}, i p_{k}\right) \sigma_{0}\right. \\
& \left.+\left[\xi_{\mathbf{k}}+\chi\left(\mathbf{k}, i p_{k}\right)\right] \sigma_{3}+\phi\left(\mathbf{k}, i p_{k}\right) \sigma_{1}\right\}, \\
\Theta\left(\mathbf{k}, i p_{k}\right)= & {\left[p_{k} Z\left(\mathbf{k}, i p_{k}\right)\right]^{2}+\left[\xi_{\mathbf{k}}+\chi\left(\mathbf{k}, i p_{k}\right)\right]^{2} } \\
& +\left[\phi\left(\mathbf{k}, i p_{k}\right)\right]^{2} .
\end{aligned}
$$

Replacing (24) and (25) by the exact forms of self-energy in (18) and (23), we come up with the Eliashberg equations,

$$
\begin{gathered}
Z\left(\mathbf{k}, i p_{k}\right)=1+\frac{T}{p_{k} N_{F}} \sum_{\mathbf{p}, n} \frac{p_{n} Z\left(\mathbf{p}, i p_{n}\right)}{\Theta\left(\mathbf{p}, i p_{n}\right)} \lambda(\mathbf{k}, \mathbf{p}, k, n), \\
\chi\left(\mathbf{k}, i p_{k}\right)=-\frac{T}{N_{F}} \sum_{\mathbf{p}, n} \frac{\xi_{\mathbf{p}}+\chi\left(\mathbf{p}, i p_{n}\right)}{\Theta\left(\mathbf{p}, i p_{n}\right)} \lambda(\mathbf{k}, \mathbf{p}, k, n),
\end{gathered}
$$

$$
\phi\left(\mathbf{k}, i p_{k}\right)=\frac{T}{N_{F}} \sum_{\mathbf{p}, n} \frac{\phi\left(\mathbf{p}, i p_{n}\right)}{\Theta\left(\mathbf{p}, i p_{n}\right)}\left[\lambda(\mathbf{k}, \mathbf{p}, k, n)-N_{F} V(\mathbf{k}-\mathbf{p})\right],
$$

where $N_{F}$ is the density of states per spin at the Fermi level and

$$
\begin{aligned}
\lambda(\mathbf{k}, \mathbf{p}, k, n) & =-N_{F} g_{\mathbf{k}-\mathbf{p}}^{2} \mathcal{P}^{0}\left(\mathbf{k}-\mathbf{p}, i p_{k}-i p_{n}\right) \\
& =\int_{0}^{\infty} \frac{2 \omega d \omega}{\left(p_{k}-p_{n}\right)^{2}+\omega^{2}} \alpha^{2} F(\mathbf{k}, \mathbf{p}, \omega)
\end{aligned}
$$

is the Eliashberg electron-bogolon spectral function [4] with

$$
\alpha^{2} F(\mathbf{k}, \mathbf{p}, \omega)=-\frac{1}{\pi} \operatorname{Im}\left[\mathcal{P}^{0}\left(\mathbf{k}-\mathbf{p}, i p_{k}-i p_{n}\right)\right] .
$$

The superconducting gap can now be found as

$$
\Delta\left(\mathbf{k}, i p_{k}\right)=\frac{\phi\left(\mathbf{k}, i p_{k}\right)}{Z\left(\mathbf{k}, i p_{k}\right)} .
$$

For the $2 \mathrm{~b}$ process, we have

$$
\alpha^{2} F(\mathbf{k}, \mathbf{p}, \omega)=\frac{N_{F} g_{\mathbf{k}-\mathbf{p}^{2}} \kappa^{2} n_{c}^{2}}{2 L^{2}} \frac{H(\omega-s|\mathbf{k}-\mathbf{p}|)}{2 \pi s^{2} \sqrt{\omega^{2}-(s|\mathbf{k}-\mathbf{p}|)^{2}}},
$$

where $H(\omega)$ is the Heaviside step function (see the details of the calculation in Appendix A).

The equation $\Delta\left(\mathbf{k}, i p_{k}\right)=0$ gives the normal-state solutions. The critical temperature $T_{c}$ can be defined as the highest temperature for which $\Delta\left(\mathbf{k}, i p_{k}\right) \neq 0$. We will use it in what follows.

\section{RESULTS}

In this section, we apply the Eliashberg theory to different hybrid systems. In particular, we consider 2DEG with the parabolic dispersion case and the electron gas with the linear dispersion case.

First of all, let us simplify the Eliashberg equations (27)-(29) using the following approximations: (i) Since the superconducting pairing mainly occurs within a narrow energy window around the Fermi surface (FS), we restrict the consideration to the electrons with $\mathbf{k}_{f}[4,44,45]$. Then, we can put $\chi\left(\mathbf{k}_{f}, i p_{k}\right)=0$ and only solve Eqs. (27) and (29); (ii) we assume the anisotropy of the Fermi surface is weak and, thus, use the isotropic formulation of the Eliashberg equations. Then, we can relabel the scalar functions (for brevity): $Z\left(\mathbf{k}=k_{f}, i p_{n}\right) \rightarrow Z_{n}$. 
In the parabolic dispersion case (and under the approximations discussed above), Eqs. (27) and (29) read [46] (see the details of the derivations in Appendix B)

$$
\begin{aligned}
Z_{n} & =1+\frac{\pi T}{p_{n}} \sum_{\nu} \frac{p_{v}}{\sqrt{p_{v}^{2}+\Delta_{v}^{2}}} \lambda(n-v), \\
Z_{n} \Delta_{n} & =\pi T \sum_{\nu} \frac{\Delta_{v}}{\sqrt{p_{v}^{2}+\Delta_{v}^{2}}}\left[\lambda(n-v)-\mu_{c}^{*}\right] .
\end{aligned}
$$

Here, we introduce the dimensionless Coulomb interaction $\mu_{c}^{*}$. By definition, it represents a double average over the FS of the term $V(\mathbf{k}-\mathbf{p})$ in (29),

$$
\mu_{c}^{*}=N_{F}\langle\langle V(\mathbf{k}-\mathbf{p})\rangle\rangle_{F S} .
$$

For large class of superconductors $[4,44,47], \mu_{c}^{*}$ is in the range of $0.1-0.2$. We will use this value instead of calculating the double-average term.

The $\lambda$ function in Eq. (34) reads

$$
\begin{gathered}
\lambda(n-v \equiv m)=\frac{\lambda_{m}}{\sqrt{1+m^{2} b_{E}^{2}}}, \\
\lambda_{m}=\frac{M^{2} s N_{F}}{16 \pi k_{f}}\left(\frac{e_{0}^{2} d}{\epsilon_{0} \epsilon}\right)^{2} \mathcal{F}\left(\arccos \phi_{0}, \frac{1}{\sqrt{1+m^{2} b_{E}^{2}}}\right), \\
b_{E}=\frac{\pi T}{s k_{f}}, \quad \phi_{0}=\frac{1}{2 k_{f} L},
\end{gathered}
$$

where $m$ is a an integer number, which indicates the difference between two Matsubara frequencies; $\mathcal{F}(\phi, m)$ is the elliptic integral of the first kind; $N_{F}=m_{e} /(2 \pi)$ with the effective mass of electron $m_{e}$; and $L$ is the effective size of the condensate introduced as the necessary cutoff.

In order to calculate $T_{c}$, we will employ the technique discussed in Ref. [46]. Since the transition temperature is defined as the point, when the energy gap is infinitesimally small, the value of $T_{c}$ can be found by setting $\Delta=0$ in all of the denominators of Eqs. (34) and (35). This gives

$$
\begin{gathered}
Z_{2 n+1}=1+\frac{1}{2 n+1} \sum_{v} \operatorname{sgn}(2 v+1) \lambda(n-v), \\
Z_{2 n+1} \Delta_{2 n+1}=\sum_{v} \frac{\Delta_{2 v+1}}{|2 v+1|}\left[\lambda(n-v)-\mu_{c}^{*}\right],
\end{gathered}
$$

where $n$ and $v$ are the Matsubara frequencies' indices, $i p_{n}=$ $\pi(2 n+1) T$, and $\operatorname{sgn}(x)$ is the signum function. Now, the equations for $Z_{2 n+1}$ are independent of the gap function $\Delta_{2 n+1}$. The critical temperature can be found by setting the determinant of the matrix to zero, det $M_{n v}=0$, where

$$
M_{n v}=\delta_{n v} Z_{2 n+1}-\frac{\lambda(n-v)-\mu_{c}^{*}}{|2 v+1|} .
$$

Furthermore, we can calculate the $T_{c}$ numerically.

Let us now consider a 2DEG with a linear dispersion in, e.g., a graphene layer [48-51]. For simplicity, we will disregard the spinor structure of the wave function assuming that we deal with the doped graphene with the Fermi energy sufficiently away from the Dirac point. Furthermore, we will disregard the contribution from different valleys $[52,53]$. This approximation is valid since the large nonzero wave-vector $\left(\sim k_{f}\right)$ strongly depresses the interaction $g_{\mathbf{p}}$ in Eq. (7).

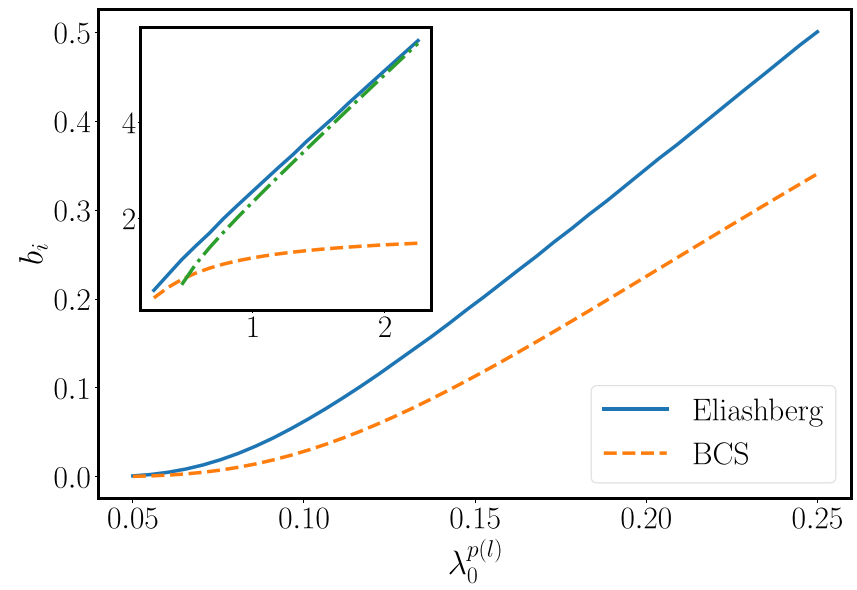

FIG. 2. Dimensionless critical temperature $b_{i}$ as a function of the dimensionless coupling constant $\lambda_{0}^{p(l)}$. The main plot corresponds to the case of small $\lambda_{0}^{p(l)}$. The inset shows the general case of arbitrary $\lambda_{0}^{p(l)}$. Blue solid line: the results of the calculation by the Eliashberg equations with $b_{\mathrm{E}}=\frac{\pi T}{s k_{f}}$; yellow dashed line: the results of the calculations by the BCS theory with $b_{\mathrm{BCS}}=\frac{\pi T}{2 \omega_{D}}$. Green dashed-dot line in the inset shows the asymptotic estimation with $b_{\mathrm{E}}=\sqrt{\left[\lambda_{0}^{p(l)} \mathcal{F}\left(\arccos \phi_{0}, 0.5\right)\right]^{2}-1}$ by Eq. (46). We used the dimensionless parameter $\phi_{0}=0.01$.

Given the assumptions discussed above, we come up with a similar system of equations as the one for the parabolic case: Eqs. (34) and (35). For the coupling constant (38) (see Appendix C), we have

$$
\lambda_{m}=\frac{M^{2} s}{32 \pi^{2} v_{f}}\left(\frac{e_{0}^{2} d}{\epsilon_{0} \epsilon}\right)^{2} \mathcal{F}\left(\arccos \phi_{0}, \frac{1}{\sqrt{1+m^{2} b_{E}^{2}}}\right),
$$

where the definitions of $b$ and $\phi_{0}$ are same as in Eq. (39).

Let us, first, understand the principle dependence of the critical temperature on the coupling constant. Comparing Eqs. (37)-(39) and (43), we see that it is convenient to denote the following dimensionless parameters:

$$
\lambda_{0}^{p(l)}=\frac{M^{2} s}{32 \pi^{2} v_{f}^{p(l)}}\left(\frac{e_{0}^{2} d}{\epsilon_{0} \epsilon}\right)^{2},
$$

where $v_{f}^{p(l)}$ is the Fermi velocity for the parabolic and linear cases, respectively. Then, we can investigate the critical temperature in terms of $b_{E}=\pi T / s k_{f}$ and $\lambda_{0}^{p(l)}$ (Fig. 2). Let us compare the critical temperatures calculated using the Eliashberg and BCS theories. In BCS, it reads

$$
T_{c}^{\mathrm{BCS}}=\frac{\gamma}{\pi} 2 \omega_{D} \exp \left(-\frac{1}{\chi}\right),
$$

where $\omega_{D}=M s^{2} / 2$ is the frequency cutoff, and $\gamma=$ $\exp C_{0}$ with $C_{0} \approx 0.577$ as Euler's constant. The parameter $\chi$ in parabolic [19] and linear [20] cases reads $\chi_{p(l)}=$ $\lambda_{0}^{p(l)} \ln \left(2 \phi_{0}^{-1}\right) / \pi$. Thus, in BCS we have a different dimensionless temperature $b_{\mathrm{BCS}}=\pi T / 2 \omega_{D}$ (compare with $b_{\mathrm{E}}$ ).

Figure 2 shows that the Eliashberg and BCS theory curves converge well when $\lambda_{0}^{p(l)}$ is small as expected; with the increase in $\lambda_{0}^{p(l)}$, then discrepancies between the two 


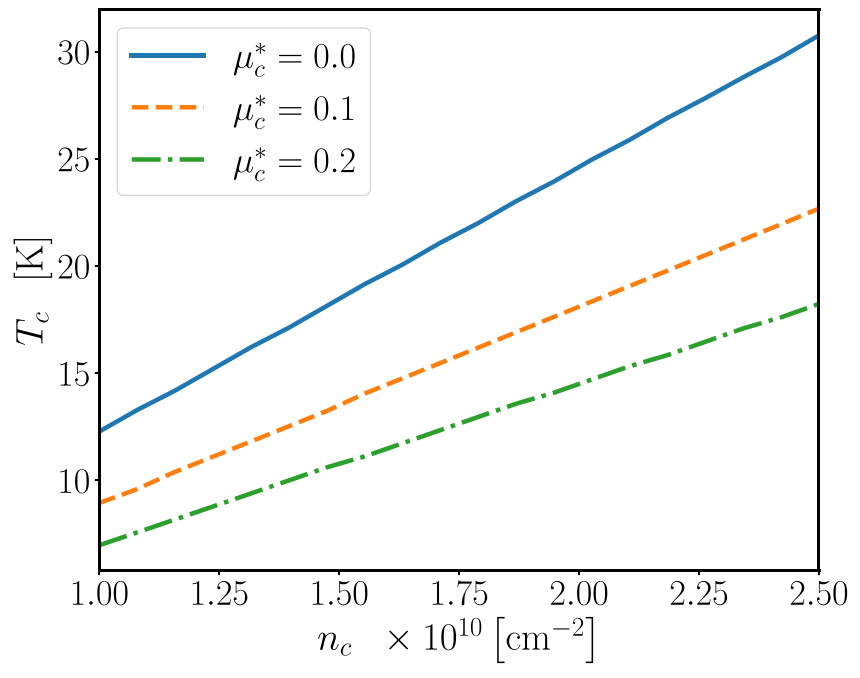

FIG. 3. The critical temperature of the SC transition as a function of condensate density calculated using the Eliashberg theory for different values of dimensionless Coulomb interaction strength $\mu_{c}^{*}$. We used the parameters typical for $\mathrm{MoS}_{2}$ : the electron effective mass $m_{e}=0.46 m_{0}$ [56] and exciton effective mass $M=$ $m_{0}$ with the free-electron mass $m_{0}$; the dielectric permittivity $\epsilon=$ 4.89; the interlayer separation $d=1.0 \mathrm{~nm}$, and the electron density $n_{e}=1.5 \times 10^{12} \mathrm{~cm}^{-2}$.

theories become more and more pronounced. As $\lambda_{0}^{p(l)} \rightarrow \infty$, the asymptotic limit gives a nearly linear dependence of the Eliashberg critical temperature on the coupling coefficient $\lambda_{0}^{p(l)}$. Such dependence is not typical for the conventional superconductors discussed in the early works $[4,46]$ in which the dependence $T_{c} \sim \sqrt{\lambda}$ in the framework of the Einstein model was found. This linear behavior can be understood as the result of the peculiarities of the angular dependence of the integral in 2D systems [54]. The nearly linear dependence which we find can be also checked analytically if we only consider the leading order of (40) and (41) (see the details in Appendix D) as the lower boundary estimation of the critical temperature $[4,46,55]$. The asymptotic behavior reads

$$
b_{E}>\sqrt{\left[\lambda_{0}^{p(l)} \mathcal{F}\left(\arccos \phi_{0}, b_{E}\right)\right]^{2}-1} .
$$

Since the elliptic integral converges faster as one increases $b_{E}$, we can treat $\mathcal{F}\left(\arccos \phi_{0}, b_{E}\right)$ as a constant. The inset plot in Fig. 2 shows an estimation of the asymptotic behavior of the dimensionless critical temperature with fixed $\mathcal{F}\left(\arccos \phi_{0}, b_{E}=0.5\right)$.

Figures 3 and 4 show the critical temperature of the SC transition as a function of condensation density. For the parabolic dispersion, we use the parameters typical for $\mathrm{MoS}_{2}$, and for the linear dispersion case we use the parameters typical for graphene.

Another interesting question is the dependence of electronbogolon interaction constant on $n_{c}$ and $n_{e}$. In the zeroth order of the electron-bogolon interaction constant, from Eq. (44) we find

$$
\begin{aligned}
& \lambda_{0}^{p} \propto \sqrt{\frac{n_{c}}{n_{e}}}, \\
& \lambda_{0}^{l} \propto \sqrt{n_{c}} .
\end{aligned}
$$

This discrepancy in the parametric dependencies can be understood by recalling the difference between the densities of states in linear and parabolic cases.

Let us address the remaining assumptions, which we used in the calculations. First, to derive Eqs. (38) and (43) in addition to the standard approximations discussed in Sec. IV, we also approximated the exciton-electron interaction by $g_{p} \approx$ $e_{0}^{2} d / 2 \epsilon_{0} \epsilon$. Such a simplification is valid if $k_{f} d$ and $k_{f} l \ll 1$. This assumption imposes a restriction on the maximal allowed value of $n_{e}$ for considered distances $d$ and $l$. If the separations $d$ and $l$ are on a nanometer scale, the density of electrons should be $n_{e} \leqslant 10^{13} \mathrm{~cm}^{-2}$.

Second, the polarization operator which we consider in Eq. (22) does not include the $N_{\mathbf{q}}$ terms as we have already mentioned. These terms are relatively small when the size of the condensate $L_{\mathrm{BEC}}$ is small as it has been shown in the analysis in the framework of the BCS theory [19,20] since these contributions give an integral truncation from $L_{\mathrm{BEC}}^{-1}$ to $k_{f} / 2$. In other words, we find the lower bound for the SC gap and the $T_{c}$. For electrons, we considered the Green's function with account of the finite temperature since we are interested in the critical temperature of the SC transition. As the earlier works on bogolon- [19,20] and phonon- [57] mediated SC point out, in the BCS theory the nonzero terms in (21) contribute to a nonmonotonous temperature dependence, and they only enhance the critical temperature. Thus, the simplifications we used are of technical nature, and they do not qualitatively change our results or conclusions.

Finally, we want to comment on the concerns regarding the applicability of our results in the context of the HohenbergMermin-Wagner theorem [58,59]. This theorem postulates a nonexistence of the long-range order in infinite 2D systems at finite temperatures. Indeed, there arise divergencies due to the presence of fluctuations. To avoid any confusion, we want to stress that in all the calculations performed in this paper we considered systems of finite size. As the result, the magnitude of the thermal fluctuations (of the phase of the

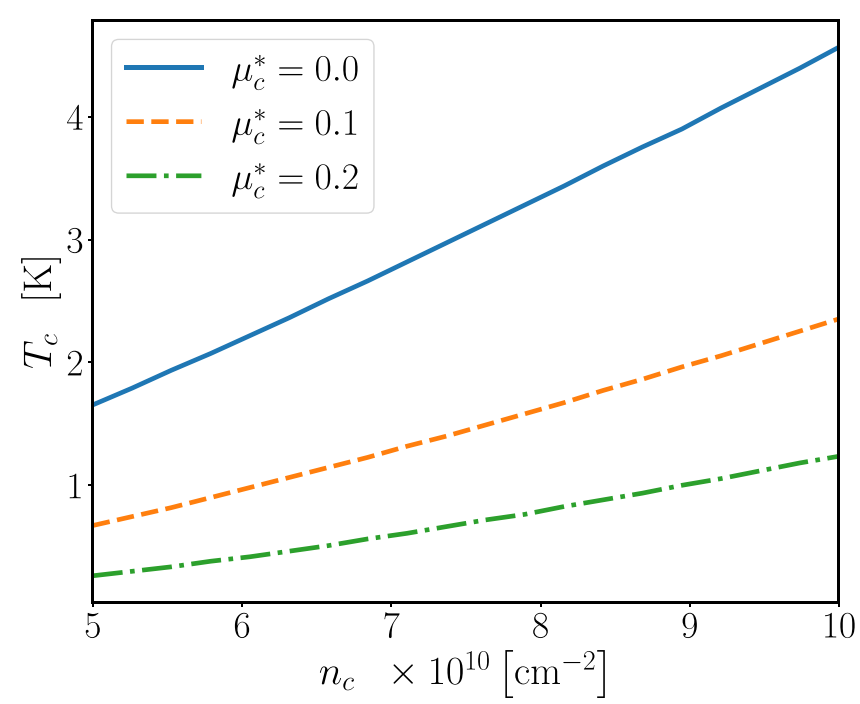

FIG. 4. Critical temperature as a function of condensate density for the linear dispersion case for different $\mu_{c}^{*}$ 's. We used $v_{f}=$ $10^{8} \mathrm{~cm} / \mathrm{s}$. Other parameters are the same as in Fig. 3 . 
order parameter) increases slowly (logarithmically) with this size, which justifies the upper-bound estimation of the critical temperature in the frameworks of the mean-field approach.

\section{CONCLUSIONS}

We developed the Eliashberg theory of the Bose condensate-mediated superconductivity in hybrid twodimensional Bose-Fermi systems and showed that bogolon-pair-mediated pairing of electrons represents the dominant mechanism not only in weak-, but also in the strong-coupling regime, whereas single-bogolon pairing is suppressed and, thus, does not play any significant role. We started with an analytic expression for the self-energy of electrons in a two-dimensional material due to their interaction with bogolons; then, we calculated the electron-bogolon coupling constant in the cases of parabolic and linear electron dispersions and presented the corresponding estimations of the critical temperature of superconducting transition, which turns out relatively high. It was demonstrated that the critical temperature of the superconducting transition depends on the dimensionless coupling constant linearly, which is not common for the conventional superconductors. We expect our theory and the estimations to impact such research areas as low-dimensional superconductors, novel two-dimensional Dirac materials, and the density functional theory for mesoscopic superconductivity.

\section{ACKNOWLEDGMENTS}

We thank Professor S. Flach and Dr. K. Villegas for useful discussions. We have been supported by the Institute for Basic Science in Korea (Project No. IBS-R024-D1) and the Russian Foundation for Basic Research (Project No. 18-29-20033).

\section{APPENDIX A: THE ELIASHBERG SPECTRAL FUNCTION}

Using the definitions Eqs. (22) and (31), we find the Eliashberg spectral function [4],

$$
\begin{aligned}
\alpha^{2} F(\mathbf{k}, \mathbf{p}, \omega) & =N_{F} g_{\mathbf{k}-\mathbf{p}}^{2}\left(-\frac{\kappa^{2} n_{c}^{2}}{2 L^{2}}\right) \int \frac{d \mathbf{q}}{(2 \pi)^{2}} \frac{-\delta\left(\omega-\omega_{\mathbf{q}+\mathbf{k}-\mathbf{p}}-\omega_{\mathbf{q}}\right)}{\omega_{\mathbf{q}+\mathbf{k}-\mathbf{p}} \omega_{\mathbf{q}}} \\
& =N_{F} g_{\mathbf{k}-\mathbf{p}}^{2}\left(-\frac{\kappa^{2} n_{c}^{2}}{2 L^{2}}\right) \mathcal{I}(\omega,|\mathbf{k}-\mathbf{p}|) .
\end{aligned}
$$

[One can easily verify Eq. (A1) by performing the integration over $\omega$ in Eq. (30).] Let us take the integral over q. For that, we denote $\mathbf{p}^{\prime}=\mathbf{k}-\mathbf{p}$ and then deal with the integral,

$$
\mathcal{I}\left(\omega, \mathbf{p}^{\prime}\right)=\int \frac{d \mathbf{q}}{(2 \pi)^{2}} \frac{(-1)}{\omega_{\mathbf{q}+\mathbf{p}^{\prime}} \omega_{\mathbf{q}}} \delta\left(\omega-\omega_{\mathbf{q}+\mathbf{p}^{\prime}}-\omega_{\mathbf{q}}\right) .
$$

By denoting $q_{1}=\left|\mathbf{q}+\mathbf{p}^{\prime}\right|$ (which we will use instead of the angle integration variable below), we find

$$
\mathcal{I}\left(\omega, \mathbf{p}^{\prime}\right)=\frac{4}{(2 \pi)^{2} s^{3}} \int_{0}^{\infty} d q \int_{\left|q-p^{\prime}\right|}^{q+p^{\prime}} d q_{1} \frac{(-1) \delta\left(\frac{\omega}{s}-q_{1}-q\right)}{\sqrt{q_{1}^{2}-\left(q-p^{\prime}\right)^{2}} \sqrt{\left(q+p^{\prime}\right)^{2}-q_{1}^{2}}} .
$$

Now, we switch the variables of integration using the substitutions $x=s\left(q+q_{1}\right)$ and $y=s\left(q_{1}-q\right)$, yielding

$$
\begin{aligned}
\mathcal{I}\left(\omega, \mathbf{p}^{\prime}\right) & =\frac{2}{(2 \pi)^{2} s^{2}} \int_{s p^{\prime}}^{\infty} \frac{d x}{\sqrt{x^{2}-s^{2} p^{\prime 2}}} \int_{-s p^{\prime}}^{s p^{\prime}} \frac{d y}{\sqrt{s^{2} p^{\prime 2}-y^{2}}}(-1) \delta(\omega-x) \\
& =\frac{2}{(2 \pi)^{2} \hbar^{3} s^{2}} \frac{(-1) H\left(\omega-\hbar s p^{\prime}\right)}{\sqrt{(\omega / \hbar)^{2}-s^{2} p^{\prime 2}}} \int_{-1}^{1} \frac{d z}{\sqrt{1-z^{2}}} \\
& =-\frac{H\left(\omega-\hbar s p^{\prime}\right)}{2 \pi \hbar^{2} s^{2} \sqrt{\omega^{2}-\left(\hbar s p^{\prime}\right)^{2}}},
\end{aligned}
$$

where $H(x)$ is the Heaviside step function. Substituting Eq. (A4) in (A1), we find the spectral function given in Eq. (33).

\section{APPENDIX B: THE PARABOLIC DISPERSION CASE}

Using the Eliashberg spectral function (33), we find

$$
\begin{aligned}
\lambda(\mathbf{k}, \mathbf{p}, n, v) & =\int_{0}^{\infty} d \omega \frac{2 \omega}{\left(p_{n}-p_{v}\right)^{2}+\omega^{2}} N_{F} g_{\mathbf{k}-\mathbf{p}}^{2} \frac{\kappa^{2} n_{c}^{2}}{2 L^{2}} \frac{H(\omega-s|\mathbf{k}-\mathbf{p}|)}{2 \pi s^{2} \sqrt{\omega^{2}-(s|\mathbf{k}-\mathbf{p}|)^{2}}} \\
& =\frac{N_{F} \kappa^{2} n_{c}^{2}}{2 \pi s^{2} L^{2}} \int_{s|\mathbf{k}-\mathbf{p}|}^{\infty} d \omega \frac{\omega g_{\mathbf{k}-\mathbf{p}}^{2}}{\left(p_{n}-p_{v}\right)^{2}+\omega^{2}} \frac{1}{\sqrt{\omega^{2}-(s|\mathbf{k}-\mathbf{p}|)^{2}}} .
\end{aligned}
$$


Again, using the notation $\mathbf{q}=\mathbf{k}-\mathbf{p}$ and noting that the integral over $\mathbf{q}$ only depends on the absolute value of $\mathbf{q}$, we find

$$
\begin{aligned}
\lambda(\mathbf{k}, \mathbf{p}, n, v) & =\frac{N_{F} \kappa^{2} n_{c}^{2}\left|g_{\mathbf{q}}\right|^{2}}{4 \pi \hbar^{2} s^{2} L^{2}} \int_{(\hbar s q)^{2}}^{\infty} \frac{d \omega^{2}}{\left(p_{n}-p_{v}\right)^{2}+\omega^{2}} \frac{1}{\sqrt{\omega^{2}-(\hbar s q)^{2}}} \\
& =\frac{N_{F} \kappa^{2} n_{c}^{2}\left|g_{\mathbf{q}}\right|^{2}}{4 \pi \hbar^{2} s^{2} L^{2}} \int_{0}^{\infty} \frac{d \Omega}{\left(p_{n}-p_{v}\right)^{2}+(\hbar s q)^{2}+\Omega} \frac{1}{\sqrt{\Omega}} .
\end{aligned}
$$

This integral is analytical (for $a b>0$ ) [60],

$$
\int \frac{d x}{(a+b x) \sqrt{x}}=\frac{2}{\sqrt{a b}} \arctan \sqrt{\frac{b x}{a}}
$$

Then, denoting $\alpha_{n v} \equiv p_{n}-p_{v}$ we find

$$
\lambda(\mathbf{k}, \mathbf{p}, n, v)=\frac{N_{F} \kappa^{2} n_{c}^{2} g_{\mathbf{k}-\mathbf{p}}^{2}}{4 s^{2} L^{2} \sqrt{\alpha_{n v}^{2}+(s|\mathbf{k}-\mathbf{p}|)^{2}}} .
$$

Furthermore, under the assumption of isotropic Fermi surface and considering the pairing to occur on the Fermi surface from Eq. (27) we find

$$
Z_{n}=1+\frac{T}{p_{n} N_{F}} \sum_{\mathbf{p}, v} \frac{p_{v} Z_{v}}{\left[p_{v} Z_{v}\right]^{2}+\xi_{\mathbf{p}}^{2}+\phi_{v}^{2}} \lambda(\mathbf{k}, \mathbf{p}, n, v)
$$

Taking $k=k_{f}$, we have

$$
Z_{n}=1+\frac{T}{p_{n} N_{F}} \sum_{v} \frac{L^{2}}{\pi^{2}} \int d p \frac{p Z_{v} p_{v}}{p_{v}^{2} Z_{v}^{2}+\xi_{p}^{2}+\phi_{v}^{2}} \int_{k_{f}-p}^{k_{f}+p} d q \frac{q}{\sqrt{q^{2}-\left(k_{f}-p\right)^{2}} \sqrt{\left(k_{f}+p\right)^{2}-q^{2}}} \frac{N_{F} \kappa^{2} n_{c}^{2} g_{q}^{2}}{4 s^{2} L^{2} \sqrt{\alpha_{n v}^{2}+(s q)^{2}}}
$$

where $\mathbf{q}=\mathbf{k}_{f}-\mathbf{p}$. Substituting $p=\sqrt{2 m_{e}(\mu+\varepsilon)}$ yields

$$
\begin{aligned}
Z_{n} & =1+\frac{T}{p_{n}} \sum_{v} \int d \varepsilon \frac{m_{e} p_{v} Z_{v}}{p_{v}^{2} Z_{v}^{2}+\xi_{p}^{2}+\phi_{v}^{2}} \int_{k_{F}-p}^{k_{F}+p} d q \frac{\frac{L^{2}}{\pi^{2}} \frac{q \kappa^{2} n_{c}^{2} g_{q}^{2}}{4 s^{2} L^{2} \sqrt{\alpha_{n v}^{2}+s^{2} q^{2}}}}{\sqrt{q^{2}-\left(k_{f}-\sqrt{k_{f}^{2}+2 m_{e} \varepsilon}\right)^{2}} \sqrt{\left(k_{f}+\sqrt{k_{f}^{2}+2 m_{e} \varepsilon}\right)^{2}-q^{2}}} \\
& \approx 1+\frac{T}{p_{n}} \sum_{v} \int d \varepsilon \frac{p_{v} Z_{v}}{p_{v}^{2} Z_{v}^{2}+\xi_{p}^{2}+\phi_{v}^{2}} \int_{k_{f}-p}^{k_{f}+p} d q \frac{q m_{e} \kappa^{2} n_{c}^{2} g_{q}^{2}}{4 s^{2} \pi^{2} \sqrt{\alpha_{n v}^{2}+s^{2} q^{2}}} \frac{1}{q \sqrt{4 k_{f}^{2}-q^{2}}},
\end{aligned}
$$

where switching to the last line in (B7) we made the perturbative expansions using $2 m_{e} \varepsilon \ll k_{f}^{2}$.

Furthermore, we can apply the residue theorem to take the integral over $\varepsilon$. Also, in the integral over $q$ we change the integral limit to $p=k_{f}$,

$$
\begin{aligned}
Z_{n} & =1+\frac{T}{p_{n}} \sum_{v} \frac{\pi p_{v}}{\sqrt{p_{v}^{2}+\Delta_{v}^{2}}} \int_{0}^{2 k_{f}} \frac{m_{e} \kappa^{2} n_{c}^{2} g_{q}^{2}}{4 s^{2} \pi^{2} \sqrt{\alpha_{n v}^{2}+s^{2} q^{2}}} \frac{d q}{\sqrt{4 k_{f}^{2}-q^{2}}} \\
& =1+\frac{T}{p_{n}} \sum_{v} \frac{p_{v}}{\sqrt{p_{v}^{2}+\Delta_{v}^{2}}} \int_{0}^{2 s k_{f}} d x \frac{m_{e} \kappa^{2} n_{c}^{2} g_{q}^{2}}{4 s^{2} \pi} \frac{1}{\sqrt{\alpha_{n v}^{2}+x^{2}}} \frac{1}{\sqrt{4 s^{2} k_{f}^{2}-x^{2}}} \\
& \approx 1+\frac{T}{p_{n}} \sum_{v} \frac{p_{v}}{\sqrt{p_{v}^{2}+\Delta_{v}^{2}}} \frac{m_{e} \kappa^{2} n_{c}^{2} g_{0}^{2}}{4 s^{2} \pi} \int_{0}^{2 s k_{f}} d x \frac{1}{\sqrt{\alpha_{n v}^{2}+x^{2}}} \frac{1}{\sqrt{4 s^{2} k_{f}^{2}-x^{2}}}
\end{aligned}
$$

where the approximation,

$$
g_{\mathbf{q}}^{2}=\frac{e_{0}^{4}(1-\exp [-q d])^{2} \exp [-2 q l]}{4 \epsilon^{2} \epsilon_{0}^{2} q^{2}} \approx \frac{e_{0}^{4} d^{2}}{4 \epsilon^{2} \epsilon_{0}^{2}} \equiv g_{0}^{2}
$$

was used. To proceed with Eq. (B8), we can use the table integral [60],

$$
\int_{u}^{b} \frac{d x}{\sqrt{\left(x^{2}+a^{2}\right)\left(b^{2}-x^{2}\right)}}=\frac{\mathcal{F}(\gamma, r)}{\sqrt{a^{2}+b^{2}}} \quad(b>u \geqslant 0),
$$


where $\mathcal{F}$ is the elliptic function of the first kind, and

$$
\gamma=\arccos \frac{u}{b}, \quad r=\frac{b}{\sqrt{a^{2}+b^{2}}} .
$$

Finally,

$$
Z_{n}=1+\frac{\pi T}{p_{n}} \sum_{v} \frac{p_{v}}{\sqrt{p_{v}^{2}+\Delta_{v}^{2}}} \lambda(n-v)
$$

Performing a similar procedure, we obtain Eq. (35).

\section{APPENDIX C: THE LINEAR DISPERSION CASE}

For the linear dispersion case, we start the derivation from Eq. (B6),

$$
Z_{n}=1+\frac{T}{p_{n} N_{F}} \sum_{v} \frac{L^{2}}{\pi^{2}} \int d p \frac{p Z_{v} p_{v}}{p_{v}^{2} Z_{v}^{2}+\xi_{p}^{2}+\phi_{v}^{2}} \int_{k_{f}-p}^{k_{f}+p} d q \frac{q}{\sqrt{q^{2}-\left(k_{f}-p\right)^{2}} \sqrt{\left(k_{f}+p\right)^{2}-q^{2}}} \frac{N_{F} \kappa^{2} n_{c}^{2} g_{q}^{2}}{4 s^{2} L^{2} \sqrt{\alpha_{n v}^{2}+(s q)^{2}}},
$$

where $\mathbf{q}=\mathbf{k}_{f}-\mathbf{p}$ and $p= \pm(\varepsilon+\mu) / v_{f}$ with $v_{f}$ as the Fermi velocity. Without the loss of generality, we assume the $n$-doping case of graphene, i.e., $\mu>0$. Then, the wave vector reads [61]

$$
p=\left\{\begin{array}{ll}
-\frac{\varepsilon+\mu}{v_{f}} & (\varepsilon<-\mu) \\
\frac{\varepsilon+\mu}{v_{f}} & (-\mu<\varepsilon)
\end{array},\right.
$$

and we have

$$
\begin{aligned}
Z_{n}= & +\frac{T}{p_{n}} \sum_{v}\left[\int_{-\mu}^{\infty} d \varepsilon\left(\frac{\varepsilon+\mu}{v_{f}^{2}}\right) \frac{p_{v} Z_{v}}{p_{v}^{2} Z_{v}^{2}+\xi_{p}^{2}+\phi_{v}^{2}} \frac{1}{\sqrt{q^{2}-\left(2 k_{f}+\frac{\varepsilon}{v_{f}}\right)^{2}} \sqrt{\left(\frac{\varepsilon}{v_{f}}\right)^{2}-q^{2}}}\right. \\
& \left.+\int_{-\infty}^{-\mu} d \varepsilon\left(\frac{\varepsilon+\mu}{v_{f}^{2}}\right) \frac{p_{v} Z_{v}}{p_{v}^{2} Z_{v}^{2}+\xi_{p}^{2}+\phi_{v}^{2}} \frac{1}{\sqrt{q^{2}-\left(\frac{\varepsilon}{v_{f}}\right)^{2}} \sqrt{\left(2 k_{f}+\frac{\varepsilon}{v_{f}}\right)^{2}-q^{2}}}\right] \frac{L^{2}}{\pi^{2}} \int_{k_{F}-p}^{k_{F}+p} d q \frac{q \kappa^{2} n_{c}^{2} g_{q}^{2}}{4 s^{2} L^{2} \sqrt{\alpha_{n v}^{2}+s^{2} q^{2}}} .
\end{aligned}
$$

Assuming $\frac{\varepsilon}{v_{f}} \ll k_{f}$ and $\frac{\varepsilon}{v_{f}} \ll q$, we can write

$$
\begin{aligned}
Z_{n} \approx & 1+\frac{T}{p_{n}} \sum_{v}\left[\int_{-\infty}^{\infty} d \varepsilon\left(\frac{\varepsilon}{v_{f}^{2}}\right) \frac{p_{v} Z_{v}}{p_{v}^{2} Z_{v}^{2}+\xi_{p}^{2}+\phi_{v}^{2}} \frac{1}{\sqrt{q^{2}\left(4 k_{f}^{2}-q^{2}\right)}}+\int_{-\infty}^{\infty} d \varepsilon\left(\frac{\mu}{v_{f}^{2}}\right) \frac{p_{v} Z_{v}}{p_{v}^{2} Z_{v}^{2}+\xi_{p}^{2}+\phi_{v}^{2}} \frac{1}{\sqrt{q^{2}\left(4 k_{f}^{2}-q^{2}\right)}}\right] \\
& \times \frac{L^{2}}{\pi^{2}} \int_{k_{f}-p}^{k_{f}+p} d q \frac{q \kappa^{2} n_{c}^{2} g_{q}^{2}}{4 s^{2} L^{2} \sqrt{\alpha_{n v}^{2}+s^{2} q^{2}}} .
\end{aligned}
$$

The integrand in the first line represents an odd function of $\varepsilon$, and, thus, it gives a vanishing contribution. For the rest terms in the equation above, we apply the residue theorem to take the integral over $\varepsilon$. Also, in the integral over $q$, we change the limit of integration to $p=k_{f}$,

$$
\begin{aligned}
Z_{n} & =1+\frac{T}{p_{n}} \sum_{v} \frac{\pi p_{v}}{\sqrt{p_{v}^{2}+\Delta_{v}^{2}}} \int_{0}^{2 k_{f}} \frac{\mu \kappa^{2} n_{c}^{2} g_{q}^{2}}{4 s^{2} \pi^{2} v_{f}^{2} \sqrt{\alpha_{n v}^{2}+s^{2} q^{2}}} \frac{d q}{\sqrt{4 k_{f}^{2}-q^{2}}} \\
& =1+\frac{T}{p_{n}} \sum_{v} \frac{p_{v}}{\sqrt{p_{v}^{2}+\Delta_{v}^{2}}} \int_{0}^{2 s k_{f}} d x \frac{\mu \kappa^{2} n_{c}^{2} g_{q}^{2}}{4 s^{2} v_{f}^{2} \pi} \frac{1}{\sqrt{\alpha_{n v}^{2}+x^{2}}} \frac{1}{\sqrt{4 s^{2} k_{f}^{2}-x^{2}}} \\
& \approx 1+\frac{T}{p_{n}} \sum_{v} \frac{p_{v}}{\sqrt{p_{v}^{2}+\Delta_{v}^{2}}} \frac{\mu \kappa^{2} n_{c}^{2} g_{0}^{2}}{4 s^{2} v_{f}^{2} \pi} \int_{0}^{2 s k_{f}} d x \frac{1}{\sqrt{\alpha_{n v}^{2}+x^{2}}} \frac{1}{\sqrt{4 s^{2} k_{f}^{2}-x^{2}}} .
\end{aligned}
$$

Using the relation given in Eqs. (B10) and (B11), we find

$$
Z_{n}=1+\frac{\pi T}{p_{n}} \sum_{v} \frac{p_{v}}{\sqrt{p_{v}^{2}+\Delta_{v}^{2}}} \frac{k_{f} \kappa^{2} n_{c}^{2} g_{0}^{2}}{4 s^{2} \pi^{2} v_{f}} \frac{1}{\sqrt{\alpha_{n v}^{2}+4 s^{2} k_{f}^{2}}} \mathcal{F}\left(\arccos \frac{1}{2 k_{f} L}, \frac{2 s k_{f}}{\sqrt{\alpha_{n v}^{2}+4 s^{2} k_{f}^{2}}}\right) .
$$


Introducing the quantities,

$$
\begin{gathered}
\lambda(n-v \equiv m)=\frac{\lambda_{m}}{\sqrt{1+m^{2} b_{E}^{2}}}, \\
\lambda_{m}=\frac{M^{2} s}{32 \pi^{2} v_{f}}\left(\frac{e_{0}^{2} d}{\epsilon_{0} \epsilon}\right)^{2} \mathcal{F}\left(\arccos \phi_{0}, \frac{1}{\sqrt{1+m^{2} b_{E}^{2}}}\right), \\
b_{E}=\frac{\pi T}{s k_{f}}, \quad \phi_{0}=\frac{1}{2 L k_{f}},
\end{gathered}
$$

we come up with a relatively simple final expression for the mass renormalization function,

$$
Z_{n}=1+\frac{\pi T}{p_{n}} \sum_{v} \frac{p_{v}}{\sqrt{p_{v}+\Delta_{v}}} \lambda(n-v) .
$$

\section{APPENDIX D: THE ASYMPTOTIC LIMIT}

Here, we will show the asymptotic limit with two different methods.

First, following the arguments discussed in Ref. [46], we can assume $b_{E}$ to be large. Then, the leading-order contribution in (37) or (43) is

$$
\lambda(0)=\lambda_{0}^{p(l)} \mathcal{F}\left(\arccos \phi_{0} \equiv \tilde{\phi}_{0}, 1\right),
$$

with zero Matsubara frequency index and we have neglected the terms $\lambda(n)$ with $|n|>1$. Then, Eqs. (40) and (41) are truncated. For Eq. (40), we find $Z_{ \pm 1}=1+\lambda(0)$, and for Eq. (41) we have $Z_{ \pm 1} \Delta_{ \pm 1}=\Delta_{ \pm 1} \lambda(0)+\Delta_{\mp 1} \lambda( \pm 1)$.

This results in a self-consistent equation,

$$
\left[\frac{\chi}{\sqrt{1+b_{E}^{2}}} \mathcal{F}\left(\tilde{\phi}_{0}, \frac{1}{\sqrt{1+b_{E}^{2}}}\right)\right]^{2}=1 .
$$

Furthermore, we can assume $\mathcal{F}\left(\tilde{\phi}_{0}, \frac{1}{\sqrt{1+b_{E}^{2}}}\right)$ to be constant for simplicity since it converges to a constant when the temperature increases as is shown in Fig. 5. Then, we find the

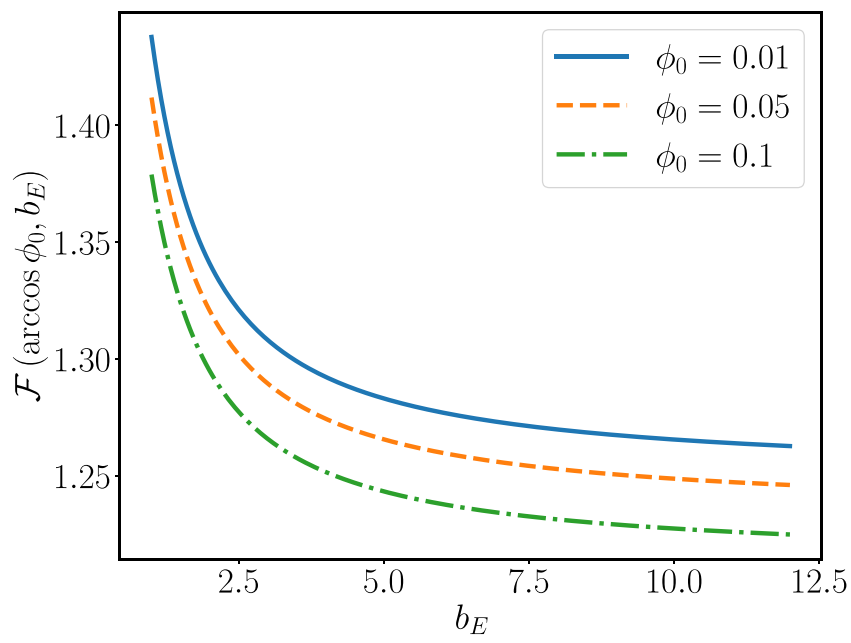

FIG. 5. Elliptic integral $\mathcal{F}\left(\arccos \phi_{0}, b_{E}\right)$ as a function of dimensionless parameter $b_{E}$. following estimation for $T_{c}$,

$$
b_{E}=\sqrt{\chi^{2} \mathcal{F}_{c}^{2}-1}
$$

where the symbol $\mathcal{F}_{c}$ means we have fixed $b_{E}$ as a constant inside $\mathcal{F}\left(\tilde{\phi}_{0}, \frac{1}{\sqrt{1+b_{E}^{2}}}\right)$.

Alternatively, we can calculate the lower boundary of critical temperature using the method introduced in Refs. [4,55]. From (33) we find

$$
\alpha^{2} F(\omega)=\sum_{\mathbf{k p}} W_{\mathbf{k}} W_{\mathbf{p}} \alpha^{2} F(\mathbf{k}, \mathbf{p}, \omega),
$$

where $W_{\mathbf{k}}=\delta\left(\varepsilon_{k}-\varepsilon_{f}\right) / N_{F}$. Considering the parabolic case, the averaged spectral function reads

$$
\alpha^{2} F(\omega)=\frac{2 s k_{f} \lambda_{0}^{p}}{\pi} \int_{0}^{2 k_{f}} \frac{H(\omega-s q) d q}{\sqrt{\left(\omega^{2}-s^{2} q^{2}\right)\left(4 k_{f}^{2}-q^{2}\right)}},
$$

where $\lambda_{0}^{p}$ is defined in (44). Then, the inequality from Ref. [55] gives

$$
1>\int_{0}^{\infty} d \omega \frac{2 \omega \alpha^{2} F(\omega)}{\left(2 \pi T_{c}\right)^{2}+\omega^{2}}
$$

where $T_{c}$ is the lower estimation of the critical temperature. The integral in (D6) gives $\left(b_{c} \equiv 2 \pi T_{c}\right)$,

$$
\begin{aligned}
\int_{0}^{\infty} & d \omega \frac{2 \omega \alpha^{2} F(\omega)}{\left(2 \pi T_{c}\right)^{2}+\omega^{2}} \\
= & \frac{2 s k_{f} \lambda_{0}^{p}}{\pi} \int_{s q}^{\infty} \frac{2 \omega d \omega}{\omega^{2}+b_{c}^{2}} \int_{0}^{2 k_{f}} \frac{d q}{\left(\omega^{2}-s^{2} q^{2}\right)\left(4 k_{f}^{2}-q^{2}\right)} \\
= & \frac{2 s k_{f} \lambda_{0}^{p}}{\pi} \int_{0}^{2 k_{f}} \frac{d q}{\sqrt{4 k_{f}^{2}-q^{2}}} \int_{0}^{\infty} \frac{d \omega}{\left(b_{c}^{2}+s^{2} q^{2}+\omega\right) \sqrt{\omega}} \\
= & 2 s k_{f} \lambda_{0}^{p} \int_{0}^{2 k_{f}} \frac{d q}{\sqrt{\left(b_{c}^{2}+s^{2} q^{2}\right)\left(4 k_{f}^{2}-q^{2}\right)}} \\
= & 2 s k_{f} \lambda_{0}^{p} \frac{\mathcal{F}(\gamma, r)}{\sqrt{4 s^{2} k_{f}^{2}+b_{c}^{2}}},
\end{aligned}
$$


where $\quad \gamma=\arccos \frac{1}{2 k_{f} L}=\arccos \phi_{0} \quad$ and $\quad r=\frac{2 s k_{f}}{\sqrt{4 s^{2} k_{f}^{2}+b_{c}^{2}}}=$ $\frac{1}{\sqrt{1+b_{E}^{2}}}$. Introducing the dimensionless parameters defined in (39), we find

$$
1>\lambda_{0}^{p} \frac{\mathcal{F}\left(\arccos \phi_{0}, \frac{1}{\sqrt{1+b_{E}^{2}}}\right)}{\sqrt{1+b_{E}^{2}}},
$$

giving

$$
b_{E}>\sqrt{\left(\lambda_{0}^{p} \mathcal{F}_{c}\right)^{2}-1}
$$

A similar routine allows to find similar dependence in the linear-dispersion case.
[1] J. Bardeen, L. N. Cooper, and J. R. Schrieffer, Microscopic theory of superconductivity, Phys. Rev. 106, 162 (1957).

[2] J. Bardeen, L. N. Cooper, and J. R. Schrieffer, Theory of superconductivity, Phys. Rev. 108, 1175 (1957).

[3] J. P. Carbotte, Properties of boson-exchange superconductors, Rev. Mod. Phys. 62, 1027 (1990).

[4] P. B. Allen and B. Mitrović, Theory of superconducting $T_{c}$, Solid State Phys. 37, 1 (1983).

[5] A. Migdal, Interaction between electrons and lattice vibrations in a normal metal, Sov. Phys. JETP 7, 996 (1958).

[6] G. M. Eliashberg, Interactions between electrons and lattice vibrations in a superconductor, Sov. Phys. JETP 11, 696 (1960).

[7] G. M. Eliashberg, Temperature Greens function for electrons in a superconductor, Sov. Phys. JETP 12, 1000 (1961).

[8] W. L. McMillan, Transition temperature of strong-coupled superconductors, Phys. Rev. 167, 331 (1968).

[9] M. Lüders, M. A. L. Marques, N. N. Lathiotakis, A. Floris, G. Profeta, L. Fast, A. Continenza, S. Massidda, and E. K. U. Gross, $A b$ initio theory of superconductivity. I. Density functional formalism and approximate functionals, Phys. Rev. B 72, 024545 (2005).

[10] M. A. L. Marques, M. Lüders, N. N. Lathiotakis, G. Profeta, A. Floris, L. Fast, A. Continenza, E. K. U. Gross, and S. Massidda, $A b$ initio theory of superconductivity. ii. application to elemental metals, Phys. Rev. B 72, 024546 (2005).

[11] A. Sanna, C. Pellegrini, and E. K. U. Gross, Combining Eliashberg Theory with Density Functional Theory for the Accurate Prediction of Superconducting Transition Temperatures and Gap Functions, Phys. Rev. Lett. 125, 057001 (2020).

[12] W. A. Little, Possibility of synthesizing an organic superconductor, Phys. Rev. 134, A1416 (1964).

[13] V. Ginzburg and D. Kirzhnits, On the problem of high temperature superconductivity, Phys. Rep. 4, 343 (1972).

[14] D. Allender, J. Bray, and J. Bardeen, Model for an exciton mechanism of superconductivity, Phys. Rev. B 7, 1020 (1973).

[15] F. P. Laussy, A. V. Kavokin, and I. A. Shelykh, ExcitonPolariton Mediated Superconductivity, Phys. Rev. Lett. 104, 106402 (2010).

[16] A. Kavokin and P. Lagoudakis, Exciton-mediated superconductivity, Nature Mater. 15, 599 (2016).

[17] P. Skopelitis, E. D. Cherotchenko, A. V. Kavokin, and A. Posazhennikova, Interplay of Phonon and Exciton-Mediated Superconductivity in Hybrid Semiconductor-Superconductor Structures, Phys. Rev. Lett. 120, 107001 (2018).

[18] F. Schlawin, A. Cavalleri, and D. Jaksch, Cavity-Mediated Electron-Photon Superconductivity, Phys. Rev. Lett. 122, 133602 (2019).

[19] M. Sun, A. V. Parafilo, K. H. A. Villegas, V. M. Kovalev, and I. G. Savenko, Theory of BCS-like bogolon-mediated superconductivity in transition metal dichalcogenides, New J. Phys. 23, 023023 (2021).

[20] M. Sun, A. V. Parafilo, K. H. A. Villegas, V. M. Kovalev, and I. G. Savenko, Bose-Einstein condensate-mediated superconductivity in graphene, 2D Mater. 8, 031004 (2021).

[21] O. Cotleţ, S. Zeytinoğlu, M. Sigrist, E. Demler, and A. Imamoğlu, Superconductivity and other collective phenomena in a hybrid Bose-Fermi mixture formed by a polariton condensate and an electron system in two dimensions, Phys. Rev. B 93, 054510 (2016).

[22] M. Matuszewski, T. Taylor, and A. V. Kavokin, Exciton Supersolidity in Hybrid Bose-Fermi Systems, Phys. Rev. Lett. 108, 060401 (2012).

[23] K. H. A. Villegas, V. M. Kovalev, F. V. Kusmartsev, and I. G. Savenko, Shedding light on topological superconductors, Phys. Rev. B 98, 064502 (2018).

[24] K. H. A. Villegas, F. V. Kusmartsev, Y. Luo, and I. G. Savenko, Optical Transistor for Amplification of Radiation in a Broadband Terahertz Domain, Phys. Rev. Lett. 124, 087701 (2020).

[25] M. V. Boev, V. M. Kovalev, and I. G. Savenko, Bogolonmediated electron capture by impurities in hybrid Bose-Fermi systems, Phys. Rev. B 97, 165305 (2018).

[26] A. Imamoğlu, R. J. Ram, S. Pau, and Y. Yamamoto, Nonequilibrium condensates and lasers without inversion: Excitonpolariton lasers, Phys. Rev. A 53, 4250 (1996).

[27] Y. E. Lozovik and V. I. Yudson, A new mechanism for superconductivity: pairing between spatially separated electrons and holes, Sov. Phys.-JETP 44, 389 (1976).

[28] M. M. Fogler, L. Butov, and K. S. Novoselov, High-temperature superfluidity with indirect excitons in van der Waals heterostructures, Nat. Commun. 5, 4555 (2016).

[29] F.-C. Wu, F. Xue, and A. H. MacDonald, Theory of twodimensional spatially indirect equilibrium exciton condensates, Phys. Rev. B 92, 165121 (2015).

[30] O. L. Berman and R. Y. Kezerashvili, High-temperature superfluidity of the two-component Bose gas in a transition metal dichalcogenide bilayer, Phys. Rev. B 93, 245410 (2016).

[31] B. Debnath, Y. Barlas, D. Wickramaratne, M. R. Neupane, and R. K. Lake, Exciton condensate in bilayer transition metal dichalcogenides: Strong coupling regime, Phys. Rev. B 96, 174504 (2017).

[32] G. Lerario, A. Fieramosca, F. Barachati, D. Ballarini, K. S. Daskalakis, L. Dominici, M. De Giorgi, S. A. Maier, G. Gigli, S. Kna-Cohen, and D. Sanvitto, Room-temperature superfluidity in a polariton condensate, Nat. Phys. 13, 837 (2017).

[33] R. Su, S. Ghosh, J. Wang, S. Liu, C. Diederichs, T. C. H. Liew, and Q. Xiong, Observation of exciton polariton condensation in a perovskite lattice at room temperature, Nat. Phys. 16, 301 (2020). 
[34] J. D. Plumhof, T. Stferle, L. Mai, U. Scherf, and R. F. Mahrt, Room-temperature Bose-Einstein condensation of cavity excitonpolaritons in a polymer, Nature Mater. 13, 247 (2014).

[35] Z. Wang, D. A. Rhodes, K. Watanabe, T. Taniguchi, J. C. Hone, J. Shan, and K. F. Mak, Evidence of high-temperature exciton condensation in two-dimensional atomic double layers, Nature (London) 574, 76 (2019).

[36] K. H. A. Villegas, M. Sun, V. M. Kovalev, and I. G. Savenko, Unconventional Bloch-Grüneisen Scattering in Hybrid BoseFermi Systems, Phys. Rev. Lett. 123, 095301 (2019).

[37] L. V. Butov, Excitonic devices, Superlattices Microstruct. 108, 2 (2017).

[38] C. Backes, A. M. Abdelkader, C. Alonso, A. Andrieux-Ledier, R. Arenal, J. Azpeitia, N. Balakrishnan, L. Banszerus, J. Barjon, R. Bartali et al., Production and processing of graphene and related materials, 2D Mater. 7, 022001 (2020).

[39] M. V. Boev, V. M. Kovalev, and I. G. Savenko, Magnetoplasmon Fano resonance in Bose-Fermi mixtures, Phys. Rev. B 94, 241408(R) (2016).

[40] M. Sun, K. H. A. Villegas, V. M. Kovalev, and I. G. Savenko, Bogolon-mediated electron scattering in graphene in hybrid Bose-Fermi systems, Phys. Rev. B 99, 115408 (2019).

[41] S. Giorgini, Damping in dilute Bose gases: A mean-field approach, Phys. Rev. A 57, 2949 (1998).

[42] Y. Nambu, Quasi-particles and gauge invariance in the theory of superconductivity, Phys. Rev. 117, 648 (1960).

[43] L. Gor'kov, On the energy spectrum of superconductors, Sov. Phys. JETP 7, 158 (1958).

[44] E. R. Margine and F. Giustino, Anisotropic Migdal-Eliashberg theory using Wannier functions, Phys. Rev. B 87, 024505 (2013).

[45] F. Schrodi, P. M. Oppeneer, and A. Aperis, Full-bandwidth Eliashberg theory of superconductivity beyond Migdal's approximation, Phys. Rev. B 102, 024503 (2020).

[46] G. D. Mahan, Many-Particle Physics (Plenum Press, New York, 1990).

[47] P. Morel and P. W. Anderson, Calculation of the superconducting state parameters with retarded electron-phonon interaction, Phys. Rev. 125, 1263 (1962).

[48] K. S. Novoselov, A. K. Geim, S. V. Morozov, D. Jiang, Y. Zhang, S. V. Dubonos, I. V. Grigorieva, and A. A. Firsov, Electric field effect in atomically thin carbon films, Science 306, 666 (2004).
[49] A. H. Castro Neto, F. Guinea, N. M. R. Peres, K. S. Novoselov, and A. K. Geim, The electronic properties of graphene, Rev. Mod. Phys. 81, 109 (2009).

[50] K. S. Novoselov, D. Jiang, F. Schedin, T. J. Booth, V. V Khotkevich, S. V. Morozov, and A. K. Geim, Two-dimensional atomic crystals, Proc. Natl. Acad. Sci. USA 102, 10451 (2005).

[51] K. S. Novoselov, A. K. Geim, S. V. Morozov, D. Jiang, M. I. Katsnelson, I. V. Grigorieva, S. V. Dubonos, and A. A. Firsov, Two-dimensional gas of massless Dirac fermions in graphene, Nature (London) 438, 197 (2005).

[52] M. Einenkel and K. B. Efetov, Possibility of superconductivity due to electron-phonon interaction in graphene, Phys. Rev. B 84, 214508 (2011).

[53] N. B. Kopnin, T. T. Heikkilä, and G. E. Volovik, Hightemperature surface superconductivity in topological flat-band systems, Phys. Rev. B 83, 220503(R) (2011).

[54] A. V. Chubukov, A. Abanov, I. Esterlis, and S. A. Kivelson, Eliashberg theory of phonon-mediated superconductivity When it is valid and how it breaks down, Ann. Phys. (NY) 417, 168190 (2020)

[55] M. V. Sadovskii, Limits of Eliashberg theory and bounds for superconducting transition temperature, Physics-Uspekhi (2021).

[56] A. Kormányos, G. Burkard, M. Gmitra, J. Fabian, V. Zólyomi, N. D. Drummond, and V. Fal'ko, $k \cdot p$ theory for twodimensional transition metal dichalcogenide semiconductors, 2D Mater. 2, 022001 (2015).

[57] N. A. Enaki and V. V. Eremeev, Cooperative two-phonon phenomena in superconductivity, New J. Phys. 4, 80 (2002).

[58] P. C. Hohenberg, Existence of long-range order in one and two dimensions, Phys. Rev. 158, 383 (1967).

[59] N. D. Mermin and H. Wagner, Absence of Ferromagnetism or Antiferromagnetism in One- or Two-Dimensional Isotropic Heisenberg Models, Phys. Rev. Lett. 17, 1133 (1966).

[60] 3-4 - definite integrals of elementary functions, in Table of Integrals, Series, and Products, eighth ed., edited by D. Zwillinger, V. Moll, I. Gradshteyn, and I. Ryzhik (Academic Press, Boston, 2015), pp. 249-519.

[61] N. B. Kopnin and E. B. Sonin, BCS Superconductivity of Dirac Electrons in Graphene Layers, Phys. Rev. Lett. 100, 246808 (2008). 